20. E. Smirnova, D. Shurland, S. Ryazantsev, A. Van Der Bliek, J. Cell Biol. 143, 351 (1998).

21. A. M. Labrousse, M. D. Zappaterra, D. A. Rube, A. M. van der Bliek, Mol. Cell 4, 815 (1999).

22. H. Sesaki, R. E. Jensen, J. Cell Biol. 147, 699 (1999).

23. S. Arimura, N. Tsutsumi, Proc. Natl. Acad. Sci. U.S.A. 99, 5727 (2002)

24. A. M. van der Bliek, Trends Cell Biol. 9, 96 (1999).

25. J. E. Hinshaw, Annu. Rev. Cell Dev. Biol. 16, 483 (2000).

26. J. E. Hinshaw, S. L. Schmid, Nature 374, 190 (1995).

27. J. E. Hinshaw, Curr. Opin. Struct. Biol. 9, 260 (1999).

28. M. H. Stowell, B. Marks, P. Wigge, H. T. McMahon, Nature Cell Biol. 1, 27 (1999).

29. B. Marks et al., Nature 410, 231 (2001).

30. N. H. Fukushima, E. Brisch, B. R. Keegan, W. Bleazard, J. M. Shaw, Mol. Biol. Cell 12, 2756 (2001).

31. S. Sever, Curr. Opin. Cell Biol. 14, 463 (2002).

32. S. L. Newmyer, A. Christensen, S. Sever, Dev. Cell 4, 929 (2003)

33. H. Gao, D. Kadirjan-Kalbach, J. E. Froehlich, K. W. Osteryoung, Proc. Natl. Acad. Sci. U.S.A. 100, 4328 (2003).

34. S. Miyagishima, K. Nishida, T. Kuroiwa, Trends Plant Sci. 8, 432 (2003).

35. S. Miyagishima et al., Plant Cell 13, 2257 (2001).

36. S. Y. Miyagishima et al., Plant Cell 15, 655 (2003).

37. K. Nishida et al., Proc. Natl. Acad. Sci. U.S.A. 100, 2146 (2003).

38. A. Legesse-Miller, R. H. Massol, T. Kirchhausen, Mol. Biol. Cell 14, 1953 (2003).

39. M. Fujiwara, S. Yoshida, Biochem. Biophys. Res. Commun. 287, 462 (2001).

40. R. S. McAndrew, J. E. Froehlich, S. Vitha, K. D. Stokes, K. W. Osteryoung, Plant Physiol. 127, 1656 (2001)

41. S. Sever, H. Damke, S. L. Schmid, J. Cell Biol. 150, 1137 (2000)

42. J. Nunnari, unpublished data.

43. A. G. McArthur et al., FEMS Microbiol. Lett. 189, 271 (2000).

44. M. Marti et al., J. Biol. Chem. 278, 24837 (2003).

45. C. A. Vater, C. K. Raymond, K. Ekena, I. Howald-Stevenson, T. H. Stevens, J. Cell Biol. 119, 773 (1992).
46. D. Hoepfner, M. van den Berg, P. Philippsen, H. F. Tabak, E. H. Hettema, J. Cell Biol. 155, 979 (2001).

47. J. Nunnari, H. Gao, K. W. Osteryoung, unpublished data.

48. F. van den Ent, L. Amos, J. Lowe, Curr. Opin. Microbiol. 4, 634 (2001)

49. K. W. Osteryoung, K. D. Stokes, S. M. Rutherford, A. L. Percival, W. Y. Lee, Plant Cell 10, 1991 (1998).

50. R. Strepp, S. Scholz, S. Kruse, V. Speth, R. Reski, Proc. Natl. Acad. Sci. U.S.A. 95, 4368 (1998).

51. K. W. Osteryoung, R. S. McAndrew, Annu. Rev. Plant Physiol. Plant Mol. Biol. 52, 315 (2001).

52. K. D. Stokes, K. W. Osteryoung, Gene 320, 97 (2003).

53. S. Vitha, R. S. McAndrew, K. W. Osteryoung, J. Cell Biol. 153, 111 (2001).

54. S. Vitha et al., Plant Cell 15, 1918 (2003).

55. R. McAndrew, C. Chi-Ham, J. E. Froehlich, K. W. Osteryoung, unpublished data.

56. A. Gaikwad, V. Babbarwal, V. Pant, S. K. Mukherjee, Mol. Gen. Genet. 263, 213 (2000).

57. M. El-Shami, S. El-Kafafi, D. Falconet, S. Lerbs-Mache, Mol. Genet. Genomics 267, 254 (2002).

58. X. Ma, W. Margolin, J. Bacteriol. 181, 7531 (1999).

59. J. Errington, R. A. Daniel, D. J. Scheffers, Microbiol. Mol. Biol. Rev. 67, 52 (2003)

60. J. Kiessling et al., J. Cell Biol. 151, 945 (2000).

61. K. D. Stokes, R. S. McAndrew, R. Figueroa, S. Vitha, K. W. Osteryoung, Plant Physiol. 124, 1668 (2000).

62. J. L. Marrison et al., Plant J. 18, 651 (1999).

63. S. Walter, J. Buchner, Angew. Chem. Int. Ed. Engl. 41, 1098 (2002).

64. O. A. Koksharova, C. P. Wolk, J. Bacteriol. 184, 5524 (2002).

65. H. Fulgosi, L. Gerdes, S. Westphal, C. Glockmann, J. Soll, Proc. Natl. Acad. Sci. U.S.A. 99, 11501 (2002).

66. J. Maple, N. H. Chua, S. G. Moller, Plant J. 31, 269 (2002).

67. K. S. Colletti et al., Curr. Biol. 10, 507 (2000).

68. R. Itoh, M. Fujiwara, N. Nagata, S. Yoshida, Plant Physiol. 127, 1644 (2001).

69. L. Griparic, A. M. van der Bliek, Traffic 2, 235 (2001).

70. E. Smirnova, L. Griparic, D. L. Shurland, A. M. van der Bliek, Mol. Biol. Cell 12, 2245 (2001).
1. K. R. Pitts, Y. Yoon, E. W. Krueger, M. A. McNiven, Mol. Biol. Cell 10, 4403 (1999).

72. S. Jakobs et al., J. Cell Sci. 116, 2005 (2003).

73. K. L. Cerveny, J. M. McCaffery, R. E. Jensen, Mol. Biol. Cell 12, 309 (2001).

74. Q. Tieu, J. Nunnari, J. Cell Biol. 151, 353 (2000).

75. P. Fekkes, K. A. Shepard, M. P. Yaffe, J. Cell Biol. 151, 333 (2000)

76. A. D. Mozdy, J. M. McCaffery, J. M. Shaw, J. Cell Biol. 151, 367 (2000).

77. Q. Tieu, V. Okreglak, K. Naylor, J. Nunnari, J. Cell Biol. 158, 445 (2002).

78. K. L. Cerveny, R. E. Jensen, Mol. Biol. Cell 14, 4126 (2003).

79. Y. Yoon, E. W. Krueger, B. J. Oswald, M. A. McNiven, Mol. Cell. Biol. 23, 5409 (2003).

80. D. I. James, P. A. Parone, Y. Mattenberger, J.-C. Martinou, J. Biol. Chem. 278, 36373 (2003).

81. H. Berman et al., Nucleic Acids Res. 28, 235 (2000).

82. PDBID: 1IYG, W. Ohashi et al., unpublished data.

83. D. Rube, S. Gandre, A. van der Bliek, 14th International C. elegans Conference, 29 June to 3 July 2003, Los Angeles, Abstract 845B.

84. W. B. Huttner, A. Schmidt, Curr. Opin. Neurobiol. 10 , 543 (2000)

85. K. Farsad et al., J. Cell Biol. 155, 193 (2001).

86. A. Schmidt et al., Nature 401, 133 (1999).

87. H. Hashimoto, Int. Rev. Cytol. 222, 63 (2003).

88. N. Garrido et al., Mol. Biol. Cell 14, 1583 (2003).

89. W. J. Jeong et al., Plant Physiol. 129, 112 (2002).

90. J. Nunnari et al., Mol. Biol. Cell 8, 1233 (1997).

91. We regret that space constraints have prevented us from citing many relevant papers. We thank $\mathrm{S}$. Merchant and $\mathrm{S}$. Miyagishima for critical reading of the manuscript; P. Beech and $A$. van der Bliek for sharing relevant findings before publication; S. Vitha, D. Yoder, S. Miyagishima, K. Naylor, A. Stone, C. Song, and H. Gao for providing images for figures; and all the members of our laboratories for invaluable contributions. Supported by NSF grants 0092448 (K.W.O.), 0313520 (K.W.O.), and 0110899 (J.N.); NIH grant R01GM62942A (J.N.); and the Michigan State University Center for Plant Products and Technologies (K.W.O.).

\title{
Cell Migration: Integrating Signals from Front to Back
}

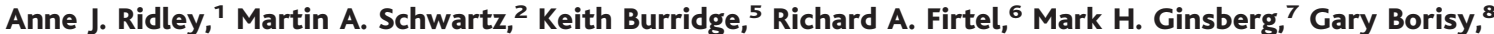 \\ J. Thomas Parsons, ${ }^{3}$ Alan Rick Horwitz ${ }^{4}$
}

Cell migration is a highly integrated multistep process that orchestrates embryonic morphogenesis; contributes to tissue repair and regeneration; and drives disease progression in cancer, mental retardation, atherosclerosis, and arthritis. The migrating cell is highly polarized with complex regulatory pathways that spatially and temporally integrate its component processes. This review describes the mechanisms underlying the major steps of migration and the signaling pathways that regulate them, and outlines recent advances investigating the nature of polarity in migrating cells and the pathways that establish it.

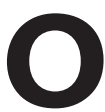

ur liaison with cell migration, as humans, begins shortly after conception, accompanies us throughout life, and often contributes to our death. Although migratory phenomena are apparent as early as implantation, cell migration orchestrates morphogenesis throughout embryonic development (1). During gastrulation, for example, large groups of cells migrate collectively as sheets to form the resulting three-layer embryo. Subsequently, cells migrate from various epithelial layers to target locations, where they then differentiate to form the specialized cells that make up different tissues and organs. Analogous migrations occur in the adult. In the renewal of skin and intestine, fresh epithelial cells migrate up from the basal layer and the crypts, respectively. Migration is also a prominent component of tissue repair and immune surveillance, in which leukocytes from the circulation mi-

${ }^{1}$ Ludwig Institute for Cancer Research, Royal Free and University College School of Medicine, London W1W 7BS, UK. ${ }^{2}$ Departments of Microbiology and Biomedical Engineering, Cardiovascular Research Center and grate into the surrounding tissue to destroy invading microorganisms and infected cells and to clear debris. The importance of cell migration however, goes far beyond humans and extends to plants and even to singlecelled organisms (2).

Migration contributes to several important pathological processes, including vascular disease, osteoporosis, chronic inflammatory diseases such as rheumatoid arthritis and multiple

Mellon Prostate Cancer Research Institute; ${ }^{3}$ Department of Microbiology; ${ }^{4}$ Department of Cell Biology; University of Virginia School of Medicine, Charlottesville, VA 22908, USA. ${ }^{5}$ Department of Cell and Developmental Biology and Lineberger Comprehensive Cancer Center, University of North Carolina, Chapel Hill, NC 27599, USA. ${ }^{6}$ Section of Cell and Developmental Biology, Division of Biological Sciences and Center for Molecular Genetics, University of California, San Diego, 9500 Gilman Drive, La Jolla, CA 92093-0634, USA. ${ }^{7}$ Department of Cell Biology, The Scripps Research Institute, 10550 North Torrey Pines Road, La Jolla, CA 92037, USA. ${ }^{8}$ Department of Cellular and Molecular Biology, Northwestern University, School of Medicine, Chicago, IL 60611, USA. 
sclerosis, cancer, and mental retardation. Thus, understanding the fundamental mechanisms underlying cell migration holds the promise of effective therapeutic approaches for treating disease, cellular transplantation, and the preparation of artificial tissues.

Over the past few years, immense progress has been made in understanding cell migration, including the establishment of polar structures, the regulation of the dynamic processes of actin and microtubule polymerization, and the regulation of spatial and temporal signal transduction. This review summarizes and highlights some of these advances in the context of the need to integrate and coordinate the many cellular events that compose migration.

\section{The Migration Cycle}

Our present understanding of cell migration is a composite derived from studies of different cell types and environments. In general, cell migration can be usefully conceptualized as a cyclic process (3). The initial response of a cell to a migration-promoting agent is to polarize and extend protrusions in the direction of migration. These protrusions can be large, broad lamellipodia or spike-like filopodia, are usually driven by actin polymerization, and are stabilized by adhering to the extracellular matrix (ECM) or adjacent cells via transmembrane receptors linked to the actin cytoskeleton. These adhesions serve as traction sites for migration as the cell moves forward over them, and they are disassembled at the cell rear, allowing it to detach. Interestingly, the movement of cell sheets shows some features of single-cell migration; however, the polarization extends across the sheet.

Although many aspects of this picture are shared among different cell types, the details can differ greatly. For example, these steps are observed most distinctly in slow-moving cells such as fibroblasts, but are not as obvious in fast-moving cells such as neutrophils, which seem to glide over the substratum. In addition, a cell's migratory behavior depends on its environment. Somitic cells migrating in vivo, for example, show large single protrusions and highly directed migration, in contrast to the multiple small protrusions they display on planar substrates; and cancer cells can modify their morphology and nature of migration in response to environmental changes $(4,5)$.

\section{The Protrusive Machinery}

Actin filaments are intrinsically polarized with fast-growing "barbed" ends and slow-growing "pointed" ends, and this inherent polarity is used to drive membrane protrusion. However, the organization of filaments depends on the type of protrusion: In lamellipodia, actin filaments form a branching "dendritic" network, whereas in filopodia they are organized into long parallel bundles (6). Actin polymerization in lamellipodia is mediated by the Arp $2 / 3$ com- plex, which binds to the sides or tip of a preexisting actin filament and induces the formation of a new daughter filament that branches off the mother filament $(6,7)$. Activation of the Arp2/3 complex is localized by WASP/WAVE family members, which are themselves activated at the cell membrane (6) (see below). Pushing of the membrane, the actual protrusive event, is believed to occur not by elongation of the actin filament per se but by an "elastic Brownian ratchet" mechanism, in which thermal energy bends the nascent short filaments, storing elastic energy. Unbending of an elongated filament against the leading edge would then provide the driving force for protrusion (7).

Several actin-binding proteins regulate the rate and organization of actin polymerization in protrusions by affecting the pool of available monomers and free ends $(7,8)$. For example, profilin prevents self-nucleation by binding to actin monomers and also serves to selectively target monomers to barbed ends. Filament elongation is terminated by capping proteins, thereby restricting polymerization to new filaments close to the plasma membrane. In addition, dis-
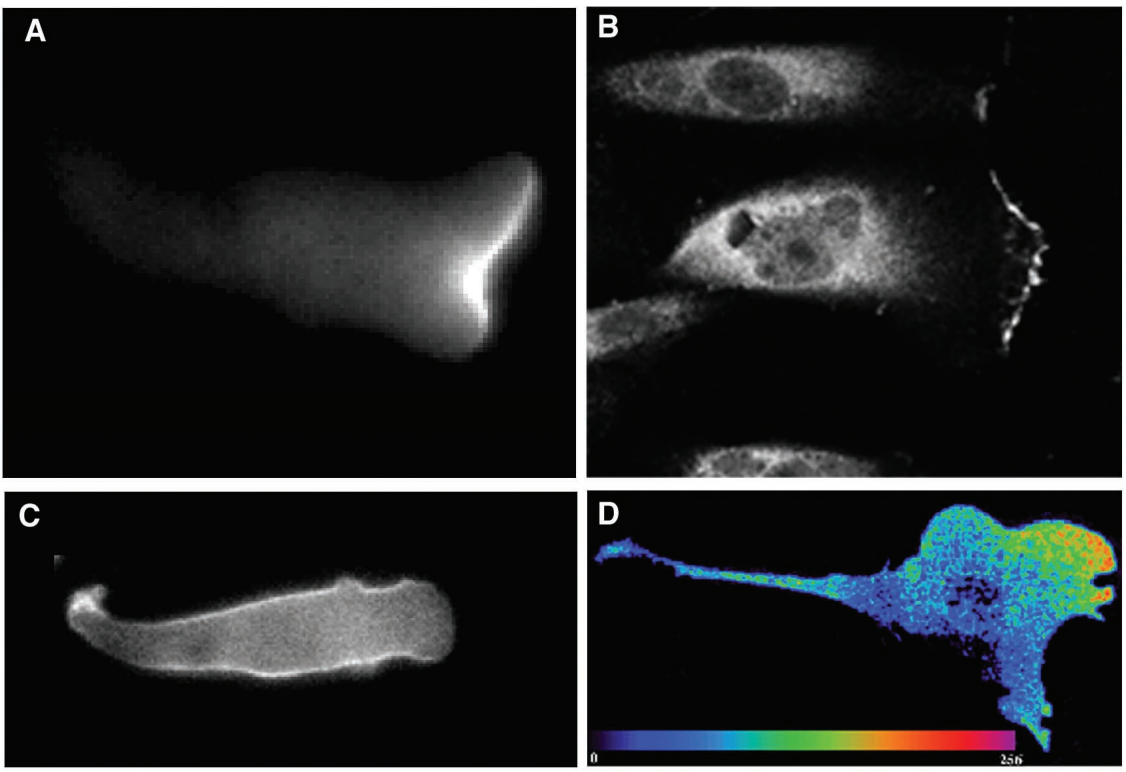

\section{direction of migration} the front end, is assisted by proteins of the $\mathrm{ADF} /$ cofilin family, which sever filaments and sissociation from the pointed end. dritic network: Cortactin stabilizes branches, whereas filamin $\mathrm{A}$ and $\alpha$-actinin stabilize the entire network by cross-linking filaments (6).

Filopodial protrusion is thought to occur which actin filaments within a bundle elongate at their barbed ends and release actin monomers from their pointed ends (6). The long and unbranched filament organization is gation rather than by branched nucleation. Many proteins are enriched at filopodial tips, including Ena/VASP proteins, which bind barbed ends of actin filaments and antagonize both capping and branching, thereby allowing continuous elongation of filaments and fascin, which bundles actin filaments and might thereby generate the stiffness needed to allow efficient pushing of the plasma membrane in filopodia (6).

Fig. 1. The polarized cell. (A) $\mathrm{PIP}_{3}$ : Leading-edge localization of a green fluorescent protein (GFP) fusion of the PH domain of Akt/PKB in chemotaxing Dictyostelium cells. (Micrograph by R. Meili and R. Firtel.) (B) Phosphorylated $\alpha 4$ integrin: Localization at the leading edge of phosphorylated $\alpha 4$ integrin expressed in migrating $\mathrm{CHO}$ cells. The localization was assayed by immunostaining with an antibody directed against the phosphorylated $\alpha 4$ integrin cytoplasmic domain. [Micrograph by L. E. Goldfinger and M. H. Ginsberg, reproduced from The Journal of Cell Biology, 2003, Vol. 162, p. 732, by copyright permission of The Rockefeller University Press] (C) PTEN: Localization of a GFP fusion of PTEN in chemotaxing Dictyostelium cells. PTEN is absent from the leading edge but present along the lateral sides and posterior of the cell. (Micrograph by R. Meili and R. Firtel.) (D) Activated Rac: Activated Rac localizes preferentially with an effector in the leading edge of migrating 3T3 cells. The interaction of GFP-V12Rac with an effector domain was assayed by fluorescence resonance energy transfer (FRET). The enhanced interaction in the leading edge is due to locally regulated membrane targeting of the V12Rac. Red and blue represent high and low intensities of FRET (that is, of interaction), respectively. (Micrograph by M. Del Pozo, W. B. Kiosses, and M. A. Schwartz.) 
The supramolecular design of lamellipodia and filopodia endows them with the capacity to perform distinct functions. Biophysical considerations suggest that the dendritic organization of lamellipodia provides a tight brush-like structure that is able to push along a broad length of plasma membrane (7). Through localized activation of the Arp $2 / 3$ complex, the lamellipodium could be induced to grow in a particular direction, providing the basis for directional migration. In contrast, filopodia, with their parallel bundle organization, are particularly well designed to serve as sensors and to explore the local environment, although they are not essential for chemotaxis.

Rho family proteins: Central regulators of protrusion. Rho family small guanosine triphosphate (GTP)-binding proteins (GTPases) are pivotal regulators of actin and adhesion organization and control the formation of lamellipodia and filopodia. They are conformationally regulated by the binding of GTP and GDP: When bound to GTP, they are active and interact with their downstream target proteins, which include protein kinases, lipid-modifying enzymes, and activators of the Arp2/3 complex (9). Rho GTPases are activated by guanine nucleotide exchange factors (GEFs) and inactivated by GTPase activating proteins (GAPs). Of the Rho GTPases, Rac, Cdc42, and RhoG are required for protrusion of lamellipodia and filopodia.

The major targets for Rac and Cdc42 that mediate actin polymerization in protrusions are the WASP/WAVE family of Arp2/3 complex activators. Rac stimulates lamellipodial extension by activating WAVE proteins (10). Cdc42 binds to WASP proteins, and in vitro this stimulates the Arp2/3 complex to induce dendritic actin polymerization (6). However, this interaction may not account for Cdc42's ability to induce filopodia, because cells lacking WASPs are still able to form filopodia (11); and, as described above, filopodia contain parallel actin filaments and not a dendritic network. RhoG does not interact directly with WASPs but appears to act upstream of Rac by binding to and activating a Rac-GEF complex (12).

WAVE/WASP proteins may themselves regulate the activity of $\mathrm{Rac}$ and $\mathrm{Cdc} 42$ by binding to GAPs and GEFs (13-15), and could thereby generate positive or negative feedback loops to regulate the extent of Cdc42/Rac-induced actin polymerization. WAVE/WASP proteins can also be regulated by other stimuli apart from $\mathrm{Cdc} 42$ and Rac, including Src family kinases, the adaptor proteins Nck and WIP, and phosphoinositides (7, 14, 16-19).

\section{Polarizing the Cell: A Keystone of Migration}

For a cell to migrate, it must be polarized, which means that the molecular processes at the front and the back of a moving cell are different. Establishing and maintaining cell polarity in response to extracellular stimuli appear to be mediated by a set of interlinked positive feedback loops involving Rho family GTPases, phosphoinositide 3-kinases (PI3Ks), integrins, microtubules, and vesicular transport (Figs. 1 and 2). Although the following discussion synthesizes information from multiple cell systems, the relative contributions of the various signals depend on the cell type and the specific stimulus.

Cdc42: A master regulator of cell polarity. $\mathrm{Cdc} 42$ is a master regulator of cell polarity in eukaryotic organisms ranging from yeast to humans. Cdc42 is active toward the front of migrating cells (20), and both inhibition and global activation of $\mathrm{Cdc} 42$ can disrupt the directionality of migration (9). One way in which $\mathrm{Cdc} 42$ influences polarity is by restricting where lamellipodia form (21) (see below). Cdc42 can also affect polarity by localizing the microtubule-organizing center (MTOC) and Golgi apparatus in front of the nucleus, oriented toward the leading edge. Cdc42-induced MTOC orientation may contribute to polarized migration by facilitating microtubule growth into the lamella and microtubule-mediated delivery of Golgiderived vesicles to the leading edge, providing membrane and associated proteins needed for forward protrusion $(9,22)$. Reorganization of the MTOC appears to be more important for the migration of slow-moving cells, because in fast-moving cells such as neutrophils and T cells, it is usually located behind the nucleus (23).

The effects of Cdc42 on MTOC position appear to be exerted mainly through a pathway involving the $\mathrm{Cdc} 42$ effector PAR6, which exists in a complex with PAR3 and an atypical protein kinase C (aPKC) (24). The molecular mechanism by which the PAR6/ PAR3/aPKC complex orients the MTOC is incompletely understood, but recent evidence suggests that it could occur as a result of local capture of microtubules at the leading edge via APC, a protein that binds tubulin and localizes to the ends of microtubules (9), via CLIP170 and IQGAP (22) and/or via the microtubule-based dynein/dynactin motor protein complex (24).

A downstream target of $\mathrm{Cdc} 42$, the kinase PAK1, can itself mediate $\mathrm{Cdc} 42$ activation downstream of heterotrimeric GTP-binding protein (G protein)-coupled receptors, which are activated by many chemoattractants. These interactions define a positive feedback loop between Cdc42 and PAK1, resulting in high Cdc42 activity at the leading edge (25). Other feedback loops involving integrins may also contribute to maintaining local Cdc42 activation $(9,26)$.

PI3Ks and PTEN: The gradient amplifiers. A surprising aspect of chemotaxis is the ability of cells to respond directionally to very shallow chemoattractant gradients (less than a 10\% dif- ference in the concentration of chemoattractant between the front and rear of a cell) (27). Such a small difference in signaling between the front and rear needs to be amplified into steeper intracellular signaling gradients in order to generate a cellular response. The phosphoinositides PtdIns $(3,4,5) \mathrm{P}_{3}\left(\mathrm{PIP}_{3}\right)$ and $\operatorname{PtdIns}(3,4) \mathrm{P}_{2}$ $\left[\mathrm{PI}(3,4) \mathrm{P}_{2}\right]$ are key signaling molecules that become rapidly and highly polarized in cells that are exposed to a gradient of chemoattractant (Fig. 2). This amplification process involves both localized accumulation and activation of PI3Ks, which generate $\mathrm{PIP}_{3} / \mathrm{PI}(3,4) \mathrm{P}_{2}$, and the phosphatase PTEN, which removes them. In Dictyostelium, for example, PI3Ks rapidly accumulate at the leading edge of cells in response to a chemoattractant, whereas PTEN becomes restricted to the sides and the rear $(27,28)$ (Fig. 1). Cells with altered PI3K or PTEN activity can usually migrate but exhibit a significantly reduced ability to move directionally up a chemoattractant gradient. Although it is not yet clear what regulates the localization of PI3Ks, Cdc42 activation is implicated in PTEN exclusion from protrusions in leukocytes, and $\mathrm{PIP}_{3}$ appears to be required for localizing $\mathrm{Cdc} 42$ activity (25). These results imply that there is a network of positive feedback loops between Cdc42, PI3K products, and PTEN that work together to initiate and maintain the polarity of migrating cells, although a $\mathrm{Cdc} 42$ paralog has not yet been identified in Dictyostelium.

Localized Rac activation: Initiating and maintaining protrusion. How do $\mathrm{Cdc} 42$ and PI3Ks lead to activation of the actin polymerization machinery required for active protrusion? The key event appears to be defining where Rac is active (Fig. 1). This is probably achieved by activating or delivering a Rac exchange factor locally, and indeed several Rac GEFs are activated by PI3K products (29). Once Rac is active, several feedback loops have been identified that help maintain directional protrusion. First, Rac can itself stimulate the recruitment and/or activation of PI3Ks at the plasma membrane, which then act upstream of Rac by $\mathrm{PIP}_{3}$-sensitive Rac GEFs $(21,29)$. Second, microtubules and Rac may form a positive feedback loop in which microtubule polymerization activates Rac, and Rac in turn stabilizes microtubules (22). Third, integrin engagement leads to Rac activation and membrane targeting (30), and so new adhesions formed at the leading edge will stimulate Rac, which in turn induces recruitment and clustering of activated integrins to the edge of lamellipodia $(31,32)$. $\mathrm{PIP}_{3}$ also contributes to integrin activation (33) and may thereby further enhance the positive feedback to Rac.

Defining the tail. Is restriction of $\mathrm{PIP}_{3}$, active $\mathrm{Cdc} 42$, and Rac to the front of the cell sufficient to make the back of the cell follow the front? In several cell types, inhibition of Rho leads to the formation of an extended tail, possibly because actomyosin-based contractility in the body of the cell is decreased. Rho may 
also act in the tail by stabilizing microtubules, which would then promote focal adhesion turnover (see below) $(22,34)$. One model for how migrating cells maintain polarity is based on the fact that Rho and Rac are mutually antagonistic, each suppressing the other's activity (35). Active Rac at the leading edge of cells would suppress Rho activity, whereas Rho would be more active at the sides and rear of the cell and suppress Rac activity, thereby preventing Rac- mediated protrusion at sites other than the leading edge $(36,37)$. However, active Rac has been implicated in detachment at the rear of migrating cells (38), and also Rho can lead to Rac activation (39).

\section{Integrins and Adhesion in Migration}

For migration to occur, a protrusion must form and then stabilize by attaching to the surroundings. Although many different re- ceptors are involved in the migration of different cell types, the integrins are a major family of migration-promoting receptors. These receptors act as the "feet" of a migrating cell by supporting adhesion to the ECM or other cells and by linking via adapters with actin filaments on the inside of the cell. As described above, integrins activate migration-related signaling molecules. They are also recipients of "inside-

A. Cell Polarization

\begin{tabular}{cc}
\multicolumn{2}{c}{ Regulators of Polarity } \\
Side/Rear & Front \\
PTEN & Activated Cdc42 \& Rac \\
Myosin II & Cdc42/PARs/aPKC \\
& PIP $3_{3}$ \\
& Activated integrin \\
& MTOC/Golgi \\
Microtubules
\end{tabular}

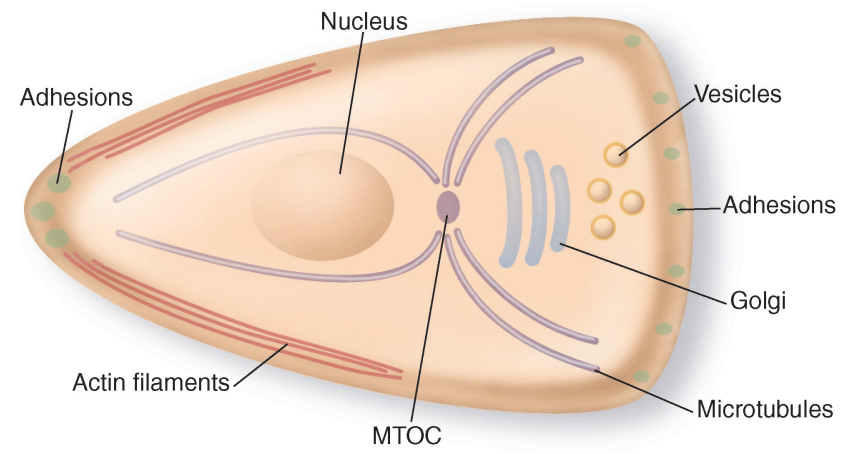

B. Protrusion and Adhesion Formation

\begin{tabular}{|cc|}
\multicolumn{2}{c|}{ Actin Polymerization } \\
Nucleation & Polymerization/Organization \\
Arp2/3 complex & Profilin \\
WAVE/WASP & ENA/VASP \\
Rac/Cdc42 & ADP/Cofilin \\
& Capping proteins \\
& Cross linkers
\end{tabular}

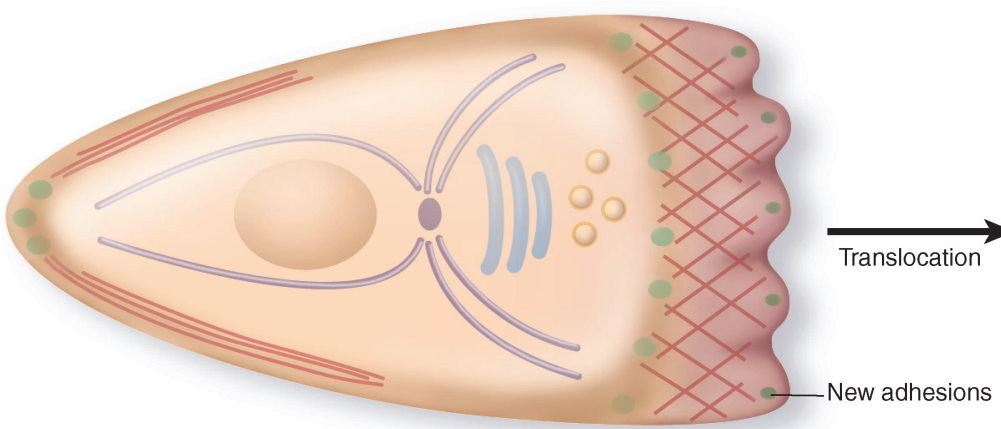

Adhesion Formation

$\begin{array}{cc}\text { Integrin Activation } & \text { Integrin Aggregation } \\ \text { Talin } & \text { Rac/Cdc42 } \\ \text { PKC } & \\ \text { Rap1 } & \\ \text { PI3K } & \end{array}$

Fig. 2. Steps in cell migration. Polarity is intrinsic to a migrating cell (A). Cdc42, along with Par proteins and aPKC, are involved in the generation of polarity. Several additional proteins are implicated in polarity, which results in directed vesicle trafficking toward the leading edge, organization of microtubules (in some cells), and the localization of the MTOC (in some cells) and Golgi apparatus in front of the nucleus. In the presence of a chemotactic agent, $\mathrm{PIP}_{3}$ is produced at the leading edge through the localized action of PI3K, which resides at the leading edge, and PTEN, a $\mathrm{PIP}_{3}$ phosphatase that resides at the cell margins and rear. PTEN and myosin II are implicated in restricting protrusions to the cell front. The migration cycle begins with the formation of a protrusion (B). WASP/ WAVE proteins are targets of Rac and Cdc42 and other signaling pathways and regulate the formation of actin branches on existing actin filaments by their action on the Arp $2 / 3$ complex. Actin polymerization, in turn, is regulated by proteins that control the availability of activated actin monomers (profilin) and debranching and depolymerizing proteins (ADF/cofilin), as well as capping and severing proteins. Protrusions are stabilized by the formation of adhesions. This process requires integrin activation, clustering, and the recruitment of structural and signaling components to nascent adhesions. Integrins are activated by talin binding and through PKC-, Rap1-, and PI3K-mediated pathways. Integrin clustering results from binding to multivalent ligands and is regulated by Rac. At the cell rear, adhesions disassemble as the rear retracts (C). This process is mediated by several possibly related signaling pathways that include Src/FAK/ERK, Rho, myosin II, calcium, calcineurin, calpain, and the delivery of components by microtubules. Many of these molecules may also regulate the disassembly of adhesions at the cell front, behind the leading edge. 
out signaling"; that is, activation to a highaffinity state by cytoplasmic signals (40).

The integrins are heterodimeric receptors consisting of $\alpha$ and $\beta$ chains with large ligand-binding extracellular domains and short cytoplasmic domains. The binding of ligands to the extracellular portion of integrins leads to conformational changes in the receptors by changing interactions between the $\alpha$ - and $\beta$-chain cytoplasmic domains (41) and to integrin clustering. This combination of occupancy and clustering initiates intracellular signals such as protein tyrosine phosphorylation, activation of small GTPases, and changes in phospholipid biosynthesis that regulate the formation and strengthening of adhesion sites, the organization and dynamics of the cytoskeleton, and cell polarity during migration (40). Although integrins themselves do not have any catalytic activity, signals are transmitted through direct and indirect interactions with many partners of integrins.

Activated integrins preferentially localize to the leading edge, where new adhesions form (31). Integrin affinity is regulated in large part by alterations in the conformation of the integrin extracellular domains that result from interactions at the integrin cytoplasmic tail (42). Activation of key intermediates such as the GTPase Rap 1 or PKC increase integrin affinity. Conversely, activation of Raf-1 kinase often suppresses integrin activation (43). The cytoskeletal linker protein talin promotes integrin activation by binding to a subset of integrin $\beta$-subunit tails and disrupting integrin $\alpha-\beta$-subunit tail interactions $(42,44)$.

The signaling potential of integrins can also be modified by posttranslational modifications of the cytoplasmic domains. For example, integrin $\alpha 4$ phosphorylation on serine blocks the binding of paxillin, a signaling adapter protein. In migrating cells, $\alpha 4$ phosphorylation at the leading edge (Fig. 1) and the consequent release of bound paxillin are required to maintain stable lamellipodia of cells migrating on ligands for integrin $\alpha 4 \beta 1$ (45).

Formation of adhesions. The mechanism by which adhesions assemble in migrating cells is a major challenge that is only beginning to be addressed. Presumably it begins with small-scale clustering due to the multivalent nature of the ECM to which the cell is adhering. Some cells, particularly rapidly migrating ones such as leukocytes, have few visible integrin clusters, and thus very small submicroscopic adhesions are probably important for their migration. In other cells, small adhesions known as focal complexes can be observed at the leading edge. Formation of these adhesions depends on $\mathrm{Rac}$ and $\mathrm{Cdc} 42$, and these adhesions stabilize the lamellipodium by mediating attachment to the ECM, thereby contributing to efficient migration. However, cells with large integrin clusters ("focal adhesions") are tightly adherent and are typically either nonmigratory or move very slowly. The assembly of focal adhesions involves Rho as well as myosin-induced contractility.

During their formation, some protein components enter adhesions with similar kinetics, which suggests that they exist in preformed cytoplasmic complexes (46). However, other components enter adhesions with very distinct kinetics, which is consistent with a model in which a regulatory event initiates the serial addition of different proteins. Paxillin, for example, is present in nascent adhesions, whereas $\alpha$-actinin appears more prominently in "older" adhesions (46).

Tractional forces. By connecting the ECM to the intracellular cytoskeleton, integrins serve as both traction sites over which the cell moves and as mechanosensors, transmitting information about the physical state of the ECM into the cell and altering cytoskeletal dynamics $(3,47,48)$. Because migrating cells must be able to detach, yet exert traction on the substratum, migration speed is a biphasic function of the strength of cell attachment. The latter is determined by the density of adhesive ligands on the substrate, the density of adhesion receptors on the cells, and the affinity of the receptors for the adhesive ligands (3). Thus, shifts in any of these parameters can have a dramatic effect on migration.

The force transmitted to sites of adhesion derives from the interaction of myosin II with actin filaments that attach to these sites. Myosin II activity is regulated by myosin light-chain (MLC) phosphorylation, which is either directly positively regulated by MLC kinase (MLCK) or Rho kinase (ROCK) or negatively regulated by MLC phosphatase, which is itself phosphorylated and inhibited by ROCK. Whereas MLCK is regulated by intracellular calcium concentration as well as by phosphorylation by a number of kinases, ROCK is regulated by binding Rho-GTP (49). MLC phosphorylation activates myosin, resulting in increased contractility and transmission of tension to sites of adhesion.

In migrating cells, the strongest forces have been reported to be transmitted to the focal complexes at the leading edge and the retracting regions at the rear (47). In contrast, in more adhesive cells, force transmitted through a focal adhesion to the substratum is proportional to the adhesion's cross-sectional area (50). It is striking that the tractional forces measured in many studies far exceed what should be needed for cell translocation. One explanation is that cells in tissue culture may be respondng to a "wound" environment, which activates Rho and thus stimulates contractility. Because traction forces are unevenly distributed over migrating cells, integrin signaling is a means of reporting these force differences to the cell.

Adhesion disassembly at the front. Adhesion disassembly is observed both at the leading edge, where it accompanies the formation of new protrusions, and at the cell rear, where it promotes tail retraction. At the front of migrating cells, adhesions at the base of a protrusion disassemble as new adhesions form at the leading edge (46). However, some adhesions persist and mature into larger, more stable structures. Little is known about adhesion disassembly versus maturation; however, targeting of microtubules has been implicated as one factor that promotes adhesion disassembly (34). Both protein kinases and phosphatases also appear to be central to the regulation of adhesion turnover and stability (51). For example, cells lacking the tyrosine kinases FAK or Src have more and larger adhesions and migrate poorly (46, 52). The interaction of FAK with Src and the adapter proteins Cas and Crk, which in turn activate Rac-specific GEFs, appears to regulate adhesion turnover. Adhesion turnover in migrating cells is also regulated by a complex of Rac-associated proteins (53) and by the mitogen-activated protein kinase ERK (54). The emerging evidence favors a model for adhesion turnover in which activation of the protein tyrosine kinases FAK and Src accompanies the formation of an adhesion signaling complex that in turn mediates the localized activation of Rac and ERK. These signals then contribute to the turnover of adhesions at the leading edge.

Adhesion disassembly and retraction at the rear. At the rear of migrating cells, adhesions must also disassemble. In fibroblasts, the rearmost adhesions often tether the cell strongly to the substratum, resulting in a long tail to the site of anchorage. The tension can be sufficient to physically break the linkage between integrin and the actin cytoskeleton, with the result that integrin is left behind while the rest of the cell moves on; a similar behavior has been observed in vivo (3). High tension exerted on the rear adhesions contributes to detachment (3). Several lines of evidence point to a role for myosin II in this event as well as in the maintenance of polarity. Dictyostelium cells deficient in myosin II or its regulator PAKa show impaired retraction and the formation of multiple pseudopodia along the sides of the cell (55). A similar phenotype is seen in monocytes or neutrophils in which myosin II assembly is blocked through inhibition of Rho or Rho kinase $(36,37)$. Al- 
though this retraction contributes to the net movement of migrating cells, it may also contribute to polarity, because the release of adhesions at the cell rear is somehow coupled to increased protrusive activity at the front.

FAK, Src, and the other regulators of adhesion turnover at the front appear to work at the rear as well. In addition, intracellular calcium levels are implicated in the disassembly of adhesions at the rear. The tension generated in migrating cells by strong adhesions in the rear can be sufficient to open stretch-activated calcium channels (56). Potential targets for calcium are the calcium-regulated phosphatase calcineurin and the calcium-activated protease calpain, which is also activated by ERK and has the potential to cleave several focal adhesion proteins, including integrins, talin, vinculin, and FAK $(57,58)$.

\section{A Molecular Model for Cell Migration}

The information discussed above can be assembled into an emerging model for how cells polarize and migrate (Fig. 2). Cells migrate directionally in response to a variety of cues, including gradients of chemokines, growth factors, or ECM molecules. These factors engage cell surface receptors, initiating a cascade of events, including the activation of $\mathrm{G}$ proteins or tyrosine kinases, the stimulation of GEFs for Cdc42, and the activation of lipid kinases and the subsequent recruitment of activated Rac. The local activation of $\mathrm{Rac}$ and/or $\mathrm{Cdc} 42$, in concert with other regulators such as WASP/WAVE family proteins and the Arp2/3 complex, stimulates the formation of a branching actin filament network at the leading edge, which in turn induces a protrusion in the direction of migration. The polymerization of actin is regulated by proteins that cap growing filaments, sever older portions of existing filaments, and control the availability of activated actin monomers. Localized activation of $\mathrm{Cdc} 42$ and Rac decreases Rho activity and enhances $\mathrm{PI} 3 \mathrm{~K}$ activity and the production of $\mathrm{PI}(3,4) \mathrm{P}_{2} / \mathrm{PIP}_{3}$ at the leading edge. Cdc42 also contributes to cell polarization by mediating reorientation of the MTOC toward the cell front, leading to growth of microtubules and delivery of vesicles into this region. Integrins and other adhesion molecules are activated by PI3Ks, PKCs, and/or Rap via talin, and they stabilize the protrusion via structural connections to the actin filaments. They also signal to Rac, which promotes recruitment of additional integrins and the formation of adhesions. New adhesions at the leading edge in turn reinforce high Rac, Cdc42, and PI3K activity, whereas the formation of a gradient of Rho activity that is low at the leading edge and higher at the rear and sides further con-
8. C. G. dos Remedios et al., Physiol. Rev. 83, 433 (2003)

9. S. Etienne-Manneville, A. Hall, Nature 420, 629 (2002).

10. G. O. Cory, A. J. Ridley, Nature 418, 732 (2002).

11. S. B. Snapper et al., Nature Cell Biol. 3, 897 (2001).

12. H. Katoh, M. Negishi, Nature 424, 461 (2003).

13. S. H. Soderling et al., Cell Biol. 4, 970 (2002).

14. G. O. Cory et al., J. Biol. Chem. 277, 45115 (2002).

15. N. K. Hussain et al., Nature Cell Biol. 3, 927 (2001).

16. S. Suetsugu et al., Dev. Cell 3, 645 (2002).

17. N. H. Higgs, T. D. Pollard, Annu. Rev. Biochem. 70, 649 (2001)

18. V. Moreau et al., Nature Cell Biol. 2, 441 (2000).

19. Y. Sasahara et al., Mol. Cell 10, 1269 (2002).

20. R. E. Itoh et al., Mol. Cell. Biol. 22, 6582 (2002).

21. S. Srinivasan et al., J. Cell Biol. 160, 375 (2003).

22. O. C. Rodriguez et al., Nature Cell Biol. 5, 599 (2003)

23. J. M. Serrador, M. Nieto, F. Sanchez-Madrid, Trends Cell Biol. 9, 228 (1999).

24. S. Etienne-Manneville, A. Hall, Curr. Opin. Cell Biol. 15, 67 (2003).

25. Z. Li et al., Cell 114, 215 (2003)

26. S. Etienne-Manneville, A. Hall, Cell 106, 489 (2001).

27. P. Devreotes, C. Janetopoulos, J. Biol. Chem. 278, 20445 (2003).

28. S. Merlot, R. A. Firtel, J. Cell Sci. 116, 3471 (2003).

29. H. C. Welch, W. J. Coadwell, L. R. Stephens, P. T. Hawkins, FEBS Lett. 546, 93 (2003).

30. M. A. DelPozo et al., EMBO J. 19, 2008 (2000)

31. W. B. Kiosses et al., Nature Cell Biol. 3, 316 (2001).

32. M. A. Schwartz, S. J. Shattil, Trends Biochem. Sci. 25, 388 (2000).

33. S. J. Shattil, Thromb. Haemostasis 74, 149 (1995).

34. J. V. Small, I. Kaverina, Curr. Opin. Cell Biol. 15, 40 (2003).

35. E. E. Evers et al., Eur. J. Cancer 36, 1269 (2000)

36. R. A. Worthylake, K. Burridge, J. Biol. Chem. 278, 13578 (2003)

37. J. Xu et al., Cell 114, 201 (2003).

38. E. M. Gardiner et al., Curr. Biol. 12, 2029 (2002).

39. T. Tsuji et al., J. Cell Biol. 157, 819 (2002).

40. B. Geiger, A. Bershadsky, R. Pankov, K. M. Yamada, Nature Rev. Mol. Cell Biol. 2, 793 (2001).

41. J. Emsley, C. G. Knight, R. W. Farndale, M. J. Barnes, R. C. Liddington, Cell 101, 47 (2000).

42. M. Kim, C. V. Carman, T. A. Springer, Science 301, 1720 (2003).

43. K. Kinbara, L. E. Goldfinger, M. Hansen, F. L. Chou, M. H. Ginsberg, Nature Rev. Mol. Cell Biol. 4, 767 (2003).

44. S, Tadokoro et al., Science 302, 103 (2003)

45. L. E. Goldfinger, J. Han, W. B. Kiosses, A. K. Howe, M. H. Ginsberg, J. Cell Biol. 162, 731 (2003).

46. D. J. Webb, J. T. Parsons, A. F. Horwitz, Nature Cell Biol. 4, E97 (2002).

47. K. A. Beningo, M. Dembo, I. Kaverina, J. V. Small, Y. L. Wang, J. Cell Biol. 153, 881 (2001).

48. C. G. Galbraith, K. M. Yamada, M. P. Sheetz, J. Cell Biol. 159, 695 (2002).

49. K. Riento, A. J. Ridley, Nature Rev. Mol. Cell Biol. 4, 446 (2003).

50. N. Q. Balaban et al., Nature Cell Biol. 3, 466 (2001).

51. M. Larsen, M. L. Tremblay, K. M. Yamada, Nature Rev. Mol. Cell Biol. 4, 700 (2003).

52. S. K. Alahari, P. J. Reddig, R. L. Juliano, Int. Rev. Cytol. 220, 145 (2002).

53. C. E. Turner, K. A. West, M. C. Brown, Curr. Opin. Cell Biol. 13, 593 (2001)

54. A. A. Brahmbhatt, R. L. Klemke, J. Biol. Chem. 278, 13016 (2003)

55. C. Y. Chung, G. Potikyan, R. A. Firtel, Mol. Cell 7, 937(2001).

S. F. Gilbert, Ed., Developmental Biology (Sinauer, Sunderland, MA, ed. 7, 2003)

2. R. S. Cotran, V. Kumar, T. Collins, S. L. Robbins, B. Schmitt, Robbins Pathologic Basis of Disease (Saunders, Philadelphia, PA, ed. 6, 1999).

3. D. A. Lauffenburger, A. F. Horwitz, Cell 84, 359 (1996).

4. B. Knight et al., Curr. Biol. 10, 576 (2000).

5. P. Friedl, K. Wolf, Nature Rev. Cancer 3, 362 (2003).

6. M. D. Welch, R. D. Mullins, Annu. Rev. Cell. Dev. Biol. 18, 247 (2000)

7. T. D. Pollard, G. G. Borisy, Cell 112, 453 (2003).
56. J. Lee, A. Ishihara, G. Oxford, B. Johnson, K. Jacobson, Nature 400, 382 (1999).

57. B. Hendey, C. B. Klee, F. R. Maxfield, Science 258, 296 (1992). Biol. 12, 46 (2002).

59. The authors apologize for omissions in citations and coverage. Strict space and citation limits prohibited us from including a complete list of appropriate citations and some of the interesting recent observations in this field.
58. A. Glading, D. A. Lauffenburger, A. Wells, Trends Cell 